\title{
Universal conductivity of graphene in the ultrarelativistic regime
}

\author{
Igor F. Herbut ${ }^{1,2}$ and Vieri Mastropietro ${ }^{3}$ \\ ${ }^{1}$ Department of Physics, Simon Fraser University, Burnaby, British Columbia, Canada V5A 1S6 \\ ${ }^{2}$ Max-Planck-Institut für Physik Komplexer Systeme, Nöthnitzer Strasse 38, 01187 Dresden, Germany \\ ${ }^{3}$ Departimento di Matematica "Federigo Enriques," Università degli Studi di Milano, Via Cesare Saldini 50, 20133 Milano, Italy
}

(Received 7 April 2013; published 31 May 2013)

\begin{abstract}
We calculate the optical $(\Lambda \gg \omega \gg T)$ conductivity in clean graphene in the ultimate low-energy regime, when retardation effects of the electromagnetic interaction become important and when the full Lorentz symmetry emerges. In contrast to what happens with the short range or with the Coulomb long-range instantaneous interactions, the optical conductivity is now no longer equal to its noninteracting value, but acquires universal corrections in powers of the fine structure constant. The coefficient of the first order correction is computed, and found to be of order one. We also present the result for the conductivity in the large- $N$ limit, with $N$ as the number of Dirac fermions species, to the order $1 / N^{2}$.
\end{abstract}

DOI: 10.1103/PhysRevB.87.205445

PACS number(s): 72.80.Vp, 73.22.Pr, 05.10.Cc

\section{INTRODUCTION}

Graphene owes several of its remarkable properties to the fact that it admits an effective relativistic quantum field theory description in terms of massless Dirac fermions in two dimensions. A dramatic manifestation of this fact is seen in its conductivity properties; recent experiments ${ }^{1}$ found that the optical conductivity in monolayer graphene is essentially constant in a wide range of frequencies, and very close to the value $(\pi / 2)\left(e^{2} / h\right)$ (that is $1 / 4$ in the natural units $e=\hbar=c=1$ which we will use), which also happens to be the value found for the system of noninteracting Dirac fermions at half filling. ${ }^{2}$ This remarkable result, however, raises a couple of natural questions of principle: why the interactions, which, at least when taken at face value, are not particularly weak in graphene, do not produce visible corrections to the noninteracting value of the conductivity? And should the conductivity, and in the optical limit in particular, in graphene be in principle equal to its noninteracting value, or are there many body corrections which may lie inside the experimental errors?

The computation of the many body interaction effects on graphene's conductivity is quite sensitive to regularizations and approximations, and several aspects of it have been controversial. In the case of short range interactions, after first perturbative computations claiming nonvanishing corrections, it was finally rigorously proved ${ }^{3}$ that there are no interaction corrections to the (zero temperature) zero frequency conductivity; all the interaction contributions to the conductivity cancel out at all orders in the renormalized expansion. The exact vanishing of interaction correction emerges as a consequence of the Ward identities and the irrelevance, in the technical renormalization group (RG) sense, of the interaction. ${ }^{3,4}$ On the other hand, in the case of long range Coulomb interactions it has been predicted that the optical conductivity is still universal and equal to $1 / 4,{ }^{5}$ the argument this time being based on the divergence of the Fermi velocity, ${ }^{6}$ and the relative (to the kinetic energy) irrelevance of the interaction. The low frequency correction to the conductivity was found to be not particularly small, due to the slow logarithmic increase of the Fermi velocity, which is what is expected in the absence of (possible) accidental cancellation. A controversy in the computation of such corrections arose in literature. ${ }^{4,5,7-9}$ Technically the reason for the controversy lies in the ambiguities produced by the ultraviolet divergences due to the continuum limit. Recently, however, the controversy has been claimed to be settled by performing a lattice computation in Ref. 10, in favor of the value originally found in Ref. 4.

On the other hand, the Fermi velocity divergence found in the Coulomb case at very low frequencies is clearly rather unphysical, and simply signals ultimate inadequacy of the usual model of instantaneous Coulomb interaction. With the increase of the Fermi velocity the retardation effects eventually become important, so that the retarded current-current interaction must be added to the Coulomb density-density interaction. Such effects have been analyzed before in Refs. 11 and 12 by a RG analysis, and it was found that the flow of the Fermi velocity stops at the velocity of light $c$, and, maybe most importantly, that the coupling constant (i.e., the charge) in the theory is exactly marginal. In particular, a lattice model for graphene interacting with an electromagnetic field was considered in Ref. 12, and by iterating the RG it was proved that the Lorentz symmetry spontaneously emerges, and that the system is asymptotically close to the so-called "reduced" quantum electrodynamics $\left(\mathrm{QED}_{4,3}\right)$.

The aim of this paper is to compute the corrections to the noninteracting value of the universal optical conductivity in the limit $\omega \gg T$, due to the full electromagnetic interaction. We show first by exact RG methods that, with the retardation effects and with the honeycomb lattice included, in units of $e^{2} / \hbar$,

$$
\sigma=\frac{N}{8}\left[1+\left(C_{1}-\frac{N}{8}\right) \frac{e^{2}}{2}+O\left(e^{4}\right)\right],
$$

where $N$ is the number of four-component Dirac fermions ( $N=2$ in graphene) and $C_{1}$ is an $N$-independent constant. In the ultimate, low-energy regime the conductivity is therefore different with respect to its noninteracting value of $1 / 4$; the dependence on the number of fermion components $N$ in the first interaction correction immediately rules out possible cancellations in general. On the other hand, the correction to conductivity, in vacuum with the dielectric constant $\epsilon=1$ assumed above, is still universal, as a consequence of the emerging Lorentz invariance, in the sense that it does not 
depend on the material parameters such as the Fermi velocity, but only on the fine structure constant.

As the lattice model for graphene becomes asymptotically close to $\mathrm{QED}_{4,3}$, which is the fixed point of the RG flow, it is worthwhile computing the optical conductivity directly in this continuum model by using standard field-theoretical methods. This way an expression identical to (1) is found, with the numerical constant $C_{1}=0.0089319$. The leading $O\left(e^{2} N\right)$ correction is identical both in the lattice and in the continuum model. In graphene, the number of Dirac fermions is $N=2$, and this correction is 0.125 and much larger than the constant $C_{1}$. In any case, as $e^{2}=4 \pi \alpha$, with $\alpha=1 / 137.036$ as the fine structure constant, in vacuum we find $\sigma / \sigma_{0}=1-0.01064$, a small correction, and at the moment within the experimental error in Ref. 1. The conductivity is therefore in principle, if not in practice, different from the noninteracting value and, because of the smallness of the fine structure constant, with a new universal value only slightly reduced from the noninteracting one. We may also observe in passing that the prefactor multiplying the charge $e^{2} / 2$ in the correction term is 0.11607 , of the same order in magnitude as found in the nonrelativistic limit for the static Coulomb interaction, ${ }^{4,5,10}$ although of the opposite sign.

Finally, possible dynamical effects of the electron spin, such as the opening of the small gap at the Dirac point due to the spin-orbit interaction, ${ }^{13}$ will in this paper, just as in all the previous work, ${ }^{11,12}$ be neglected.

\section{RENORMALIZATION GROUP COMPUTATION}

We can describe graphene by a system of electrons on the honeycomb lattice interacting with an electromagnetic field. The Hamiltonian is $H=H_{e}+H_{a}$, where

$$
H_{e}=-t \sum_{\substack{\vec{x} \in \Lambda \\ j=1,2,3}} \sum_{\sigma=1}^{N} a_{\vec{x}, \sigma}^{+} b_{\vec{x}+\vec{\delta}_{i}, \sigma}^{-} e^{i e \int_{0}^{1} \vec{\delta}_{j} \vec{a}\left(\vec{x}+s \vec{\delta}_{j}, 0\right)}+\text { c.c., }
$$

with $\Lambda=\left(n_{1} \vec{l}_{1}+\vec{n}_{2} \vec{l}_{2}\right), \quad n_{i}=0, \ldots, L-1, \quad$ and $\quad \vec{l}_{1,2}=$ $\frac{1}{2}(3, \pm \sqrt{3}), \vec{\delta}_{1}=(1,0), \vec{\delta}_{2}=\frac{1}{2}(-1, \sqrt{3}), \vec{\delta}_{3}=\frac{1}{2}(-1,-\sqrt{3})$, and $H_{a}$ is the free photon Hamiltonian.

The physical observables are conveniently obtained in terms of the following generating functional for the external source fields $A$ and $\lambda$ :

$$
e^{W_{L, \beta}(A, \lambda)}=\int P(d \psi) \int P(d a) e^{\mathcal{V}(a+A, \psi)+(\psi, \lambda)},
$$

where $\psi_{\mathbf{x}}^{ \pm}=\left(a_{\mathbf{x}, \sigma}^{ \pm}, b_{\mathbf{x}+\delta_{1}, \sigma}^{ \pm}\right)$are Grassman variables (denoted with a slight abuse of notation with the same symbol), $\sigma=1, \ldots, N, \delta_{j}=\left(0, \vec{\delta}_{j}\right), P(d \psi)$ is the fermionic integration with propagator,

$$
g(\mathbf{x}-\mathbf{y})=\frac{1}{\beta|\Lambda|} \sum_{\mathbf{k} \in \mathcal{D}} e^{i \mathbf{k}(\mathbf{x}-\mathbf{y})}\left(\begin{array}{cc}
i k_{0} & v \Omega^{*}(\vec{k}) \\
v \Omega(\vec{k}) & i k_{0}
\end{array}\right)^{-1},
$$

with $\mathbf{x}=\left(x_{0}, \vec{x}\right), \mathbf{k}=\left(k_{0}, \vec{k}\right), k_{0}=\frac{2 \pi}{\beta}(m+1 / 2), \vec{k}=\frac{m_{1}}{L} \vec{b}_{1}+$ $\frac{m_{2}}{L} \vec{b}_{2}, \vec{b}_{1,2}=\frac{2 \pi}{3}(1, \pm \sqrt{3}), 0 \leqslant m_{i}<L,|\Lambda|=L^{2}, v=\frac{3}{2} t$, and $\Omega(\vec{k})=\frac{2}{3} \sum_{j=1,2,3} e^{i \vec{k}\left(\vec{\delta}_{j}-\vec{\delta}_{1}\right)}$. In the limit $L \rightarrow \infty \frac{1}{|\Lambda|} \sum_{\vec{k}} \rightarrow$ $S \int_{\mathcal{B}} \frac{d \vec{k}}{(2 \pi)^{2}}, S=\frac{3 \sqrt{3}}{2}$ is the area of the hexagonal cell and the integral is over the Brillouin zone. The dispersion relation $\Omega(\vec{k})$ vanishes at the two Fermi points $\mathbf{k}_{F}^{ \pm}=\left(0, \frac{2 \pi}{3}, \pm \frac{2 \pi}{3 \sqrt{3}}\right) . a_{\mu, \mathbf{x}}$ is a Gaussian variable while $P(d a)$ is the photon integration with propagator $\delta_{\mu, \nu} w(\mathbf{x}-\mathbf{y})$ and $\widehat{w}(\mathbf{p})=\frac{1}{S} \frac{\chi(\mathbf{p})}{2|\mathbf{p}|}$, where $\chi(\mathbf{p})$ is a cutoff function and the Feynman gauge is assumed. Finally, the interaction $\mathcal{V}$ can be easily deduced from (2) and it has the form

$$
V(a, \psi)=\sum_{\mu=1,2,3} e \int d \mathbf{x}\left(a_{0, \mathbf{x}} j_{0, \mathbf{x}}+v \vec{a}_{\mathbf{x}} \vec{j}_{\mathbf{x}}\right)+F(a),
$$

where $F(a)$ contains a higher order term in $a, j_{\mu, \mathbf{x}}=\left(j_{0, \mathbf{x}}, \vec{j}_{\mathbf{x}}\right)_{\mu}$, with

$$
\begin{aligned}
j_{0, \mathbf{x}} & =\sum_{\sigma=1}^{N}\left[a_{\mathbf{x}, \sigma}^{+} a_{\mathbf{x}, \sigma}^{-}+a_{\mathbf{x}+\delta_{1}, \sigma}^{+} a_{\mathbf{x}+\delta_{1}, \sigma}^{-}\right], \\
\vec{j}_{\mathbf{x}} & =\frac{2}{3} \sum_{j=1,2,3} \sum_{\sigma=1}^{N} \vec{\delta}_{j}\left(a_{\mathbf{x}, \sigma}^{+} b_{\mathbf{x}+\delta_{j}, \sigma}^{-}-b_{\mathbf{x}+\delta_{j}, \sigma}^{+} a_{\mathbf{x}, \sigma}^{-}\right) .
\end{aligned}
$$

From the identity $W_{L, \beta}(A, \lambda)=W_{L, \beta}\left(A+\partial \alpha, \lambda e^{i \alpha_{\mathbf{x}}}\right)$, we get

$$
\frac{\partial}{\partial \alpha} W_{L, \beta}\left(A+\partial \alpha, \lambda e^{i \alpha_{\mathbf{x}}}\right)=0
$$

and the derivatives of the above relations provide a set of Ward identities. Calling $K_{\mu, v}(\mathbf{p})=\left.\frac{1}{S} \frac{\partial^{2} W_{L, \beta}(A, 0)}{\partial \widehat{A}_{\mu, \mathbf{p}} \partial \widehat{A}_{v,-p}}\right|_{0}, \mathbf{p}=(\omega, p)$, the zero-temperature conductivity, at zero frequency, is defined via Kubo formula $\sigma=\lim _{\omega \rightarrow 0}-e^{2} \frac{1}{\omega} K_{i, i}(\omega, 0)$. Note that $K_{i, j}(\mathbf{p})$ is the sum of the truncated current-current correlation and of the diamagnetic term, which is a constant in $\mathbf{p}$; by the Ward identity obtained from (7) with a derivative with respect to $A$, we get $\left.\lim _{p_{1} \rightarrow 0} \lim _{\omega \rightarrow 0} \widehat{K}_{11}(\mathbf{p})\right|_{p_{2}=0}=$ $\lim _{p_{1} \rightarrow 0} \lim _{\omega \rightarrow 0} \frac{\omega}{p_{1}} \widehat{K}_{01}(\mathbf{p})=0$ and, as $\widehat{K}_{11}(\mathbf{p})$ is continuous at weak coupling, we can reverse the limits so that the conductivity can be written as

$$
\sigma=\lim _{\omega \rightarrow 0^{+}}-e^{2} \frac{\widehat{K}_{i i}(\omega, 0)-\widehat{K}_{i i}(0,0)}{\omega} .
$$

There is therefore no need of computing the diamagnetic term, but it is sufficient to compute the current-current correlation and subtract the value in $\mathbf{p}=(0,0)$.

$K_{i, j}(\mathbf{p})$ can be computed by Wilsonian RG; for details, see Ref. 12. The starting point consists of writing the Grassman variables as sums of variables with momenta closer and closer to the two Fermi points $\mathbf{k}_{F}^{ \pm}=\left(0, \frac{2 \pi}{3}, \pm \frac{2 \pi}{3 \sqrt{3}}\right)$, that is

$$
\widehat{\psi}=\psi^{(1)}+\sum_{\varepsilon= \pm} \sum_{h=-\infty}^{0} \psi_{\varepsilon}^{(h)}
$$

where $\psi_{\varepsilon}^{(h)}$ lives on a shell of momenta distant $O\left(2^{h}\right)$ from $\mathbf{k}_{F}^{\varepsilon}$. Similarly, we can write $a^{\mu}=\sum_{h=-\infty}^{0} a_{h}^{\mu}$, and $a_{h}^{\mu}$ has propagator $f_{h}(\mathbf{k}) w(\mathbf{k})$ with $f_{h}(\mathbf{k})$ nonvanishing for $2^{h-1} \leqslant|\mathbf{k}| \leqslant 2^{h+1}$. After the integration of the fields $\left(\psi^{1}, a^{1}\right), \ldots,\left(\psi^{h}, a^{h}\right)$, one finds that the generating functional can be written as

$$
\begin{aligned}
e^{W_{L, \beta}(A, \lambda)}= & e^{B^{h}(A, \lambda)} \int \prod_{\varepsilon= \pm 1} P\left(d \psi_{\varepsilon}^{(\leqslant h)}\right) \\
& \times \int P\left(d a^{(\leqslant h)}\right) e^{V^{h}\left(\sqrt{Z_{h}} \psi^{(\leqslant h)}, a^{(\leqslant h)}+A, \lambda\right)},
\end{aligned}
$$


where $P\left(d \psi_{\varepsilon}^{(h)}\right)$ has the propagator

$$
g_{\varepsilon}^{(h)}\left(\mathbf{k}^{\prime}+\mathbf{k}_{F}^{\varepsilon}\right)=\frac{1}{Z_{h}}\left(\begin{array}{cc}
i k_{0} & v_{h} \Omega^{*}(\vec{k}) \\
v_{h} \Omega(\vec{k}) & i k_{0}
\end{array}\right)^{-1} .
$$

$Z_{h}$ is the wave function renormalization and $v_{h}$ is the effective Fermi velocity. Note also that (12) can be written as

$$
g_{\varepsilon}^{(h)} \sim \frac{1}{Z_{h}}\left(\begin{array}{cc}
i k_{0} & v_{h}\left(-i k_{1}^{\prime}+\varepsilon k_{2}\right) \\
v_{h}\left(i k_{1}^{\prime}+\varepsilon k_{2}\right) & i k_{0}
\end{array}\right)^{-1},
$$

so that it becomes ever closer to the Dirac propagator. By power counting, the scaling dimension of the interactions is $D=3-n$ if $n$ is the number of fields; the local terms quadratic in $a$, describing the photon mass, are absent by exploiting the Ward identity generated by (7) (see Appendix E1 of Ref. 12 for an explicit computation at one loop), while the marginal terms $a^{+} \partial a$ are vanishing by symmetry; in the same way the local terms $\psi^{+} \psi$ are vanishing by parity, and $\psi^{+} \partial \psi$ contribute to the wave function renormalization and the Fermi velocity. The effective potential is given by (at $\lambda=0$ for definiteness)

$$
V^{h}\left(\sqrt{Z_{h}} \psi^{\leqslant h}, a+A, 0\right)=\sum_{\mu} \sum_{\mathbf{x}} Z_{\mu, h}\left(a_{\mu, \mathbf{x}}^{\leqslant h}+A_{\mu, \mathbf{x}}\right) j_{\mu, \mathbf{x}}^{\leqslant h}+F_{h},
$$

where $F_{h}$ are the irrelevant terms, with negative dimension $D<0$.

From the Ward identities (7) we get

$$
\frac{Z_{0, h}}{Z_{h}}=1+O\left(e^{2}\right), \quad \frac{Z_{i, h}}{Z_{h} v_{h}}=1+O\left(e^{2}\right) .
$$

Moreover, by symmetry $Z_{1, h}=Z_{2, h}$ and by an explicit computation,

$$
Z_{h} \sim 2^{-\eta h}, \quad v_{h} \sim 1+A(1-v) 2^{\widetilde{\eta} h},
$$

with $\eta, \tilde{\eta}>0$ and $O\left(e^{2}\right)$ and $A$ is a bounded function. Therefore, the wave function renormalization is diverging (i.e., there is an anomalous dimension), and by iterating the RG the Fermi velocity converges to the velocity of light.

The model considered in Ref. 11 therefore emerges naturally starting from the lattice model. Moreover, the Lorentz symmetry is restored in the infrared limit, as the Fermi velocity flows up to the velocity of light (which in our units is 1 ). The effective couplings $e_{h, 0}=e \frac{Z_{0, h}}{Z_{h}}$ and $e_{h, i}=e \frac{Z_{1, h}}{Z_{h} v_{h}}$ flow to a line of fixed points

$$
e_{h, i} \rightarrow e+O\left(e^{3}\right)
$$

that is, the theory is exactly marginal. One obtains then a renormalized expansion for the current-current correlations in terms of the effective couplings $e_{\mu, h}$, namely $\sigma=\sigma^{(0)}+$ $\sigma^{(2)}+\cdots$. This differs from perturbative expansion in that there are no infrared divergences and all coefficients are bounded. We get

$$
\begin{aligned}
\sigma^{(0)}= & N e^{2} \lim _{\omega \rightarrow 0^{+}} \sum_{\varepsilon= \pm} \frac{1}{\omega} \sum_{h \leqslant 1} \int \frac{d k_{0}}{2 \pi} \int_{\mathcal{B}} \frac{d \vec{k}^{\prime}}{(2 \pi)^{2}} \frac{\left(Z_{1, h}\right)^{2}}{Z_{h} Z_{h}} \\
& \times \operatorname{Tr}\left\{\sigma_{1} g_{\varepsilon}^{(h)}\left(\mathbf{k}^{\prime}\right) \sigma_{1}\left[g_{\varepsilon}^{(h)}\left(\mathbf{k}^{\prime}+(\omega, \overrightarrow{0})\right)-g_{\varepsilon}^{(h)}\left(\mathbf{k}^{\prime}\right)\right]\right\},
\end{aligned}
$$

where $\sigma_{j}$ are Pauli matrices and $N=2$ in graphene. Note the presence of a factor $\frac{1}{Z_{h}}$ for any fermionic line, and of a factor $Z_{i, h}$ for any vertex. The presence of such factors could radically alter the conductivity properties; for instance, if we do not take into account the vertex renormalization, that is we replace $Z_{i, h}$ with unity, one would get $\sigma^{(0)}=0$. On the contrary, thanks to the Ward identity and Eq. (14) we can replace $\frac{\left(Z_{h}^{(i)}\right)^{2}}{Z_{h} Z_{h}}$ with $v_{h}$ up to $O\left(e^{2}\right)$ terms. The integral then still appears to be nonuniversal, as it depends of the effective Fermi velocity and on the lattice details. However, it is not so; we can write an integral over the Brillouin zone $\mathcal{B}$ as the sum of two terms, one $|\Omega(\vec{k})| \geqslant \varepsilon$ and the other $|\Omega(\vec{k})| \leqslant \varepsilon$; the former is uniformly convergent as $\omega \rightarrow 0^{+}$: therefore, we can exchange the integral with the limit and check that the integral of the limit is zero simply because the integrand is odd in $k_{0}$. In the latter we use (12) neglecting the corrections (as the size of the integral is arbitrarily small); the dependence from $v_{h}$ disappears through a change of variables and we finally get $\sigma^{(0)}=e^{2} \frac{N}{8}+O\left(N e^{4}\right)$. Moreover, as shown in Ref. 14, the low frequency corrections are $O\left(e^{2} \omega^{2}\right)$.

Let us consider now $\sigma^{(2)}$; there are three possible contributions, $\sigma^{(2)}=\sigma_{a}^{(2)}+\sigma_{b}^{(2)}+\sigma_{c}^{(2)}$, but only one of them, which we call $\sigma_{c}^{(2)}$, is proportional to $N^{2}$. Therefore, if we find it to be nonvanishing we can safely conclude that the dc conductivity is different from the noninteracting value, at least for a generic $N$. The value of such a term is

$$
\begin{aligned}
\sigma_{c}^{(2)}= & -N^{2} e^{4} \lim _{\omega \rightarrow 0^{+}} \frac{1}{2}\left\{\frac{1}{\omega} \sum_{\varepsilon= \pm} \sum_{h(\omega) \leqslant h \leqslant 1} \int \frac{d k_{0}}{2 \pi} \int_{\mathcal{B}} \frac{d \vec{k}^{\prime}}{(2 \pi)^{2}} \frac{\left(Z_{1, h}\right)^{2}}{Z_{h} Z_{h}}\right. \\
& \left.\times \operatorname{Tr}\left\{\sigma_{1}\left(\vec{k}^{\prime}\right) g_{\varepsilon}^{(h)}\left(\mathbf{k}^{\prime}\right) \sigma_{1}\left[g_{\varepsilon}^{(h)}\left(\mathbf{k}^{\prime}+(\omega, \overrightarrow{0})\right)-g_{\varepsilon}^{(h)}\left(\mathbf{k}^{\prime}\right)\right]\right\}^{2}\right\},
\end{aligned}
$$

where $h(\omega)$ is such that $2^{h(\omega)} \sim|\omega|$. In writing the above expression we have used that only the renormalized parts of the "bubble" diagrams contribute, as their local part is vanishing. Again using that $Z_{i, h} / Z_{h}=v_{h}+O\left(e^{2}\right)$ we get $\sigma_{c}^{(2)}=-\left(e^{4} / 2\right)(N / 8)^{2}+O\left(e^{4} N\right)$, and finally (1) is found. The $N$ dependence allows us to exclude the possibility of cancellations, and we can conclude that the optical conductivity is different from its noninteracting value, with a leading correction which is universal. Note the difference with the case of Coulomb interactions: in such a case at one loop $v_{h} \sim 1 /|h|$, while $Z_{h}, Z_{i, h}, e_{i, h}$ are essentially constants so that $\sigma_{c}^{(2)}$ vanishes. Similarly, for Hubbard interactions the photon propagator $1 / 2|\omega|$ should be replaced by a constant, and again $\sigma_{c}^{(2)}$ would be vanishing.

\section{EFFECTIVE QED DESCRIPTION}

The previous analysis shows that the lattice graphene system flows, by iterating the RG, to a fixed point expressed by the continuum $\mathrm{QED}_{4,3}$. It is useful then to study the conductivity properties in the continuum theory (assuming as usual that the value of the conductivity depends only on the fixed point of the $\mathrm{RG}^{15}$ ) in order to get more information on the subleading corrections. The action for the effective model 
in $2+1$ dimensions is

$$
\begin{aligned}
S= & \int d \mathbf{x}\left[\bar{\Psi}_{i}(\mathbf{x}) \gamma_{\mu}\left\{\partial_{\mu}-e\left[a_{\mu}(\mathbf{x})-A_{\mu}(\mathbf{x})\right]\right\} \Psi_{i}(\mathbf{x})\right. \\
& \left.+\frac{1}{2} \int d \mathbf{x} d \mathbf{y} W_{\mu \nu}^{-1}(\mathbf{x}-\mathbf{y}) a_{\mu}(\mathbf{x}) a_{\nu}(\mathbf{y})\right],
\end{aligned}
$$

where $\Psi$ is a four-component fermionic field, $i=1, \ldots, N$, $\mathbf{x}=\left(x_{0}, x_{1}, x_{2}\right)$, and the summation convention is assumed. Note that $\vec{x}=\left(x_{1}, x_{2}\right)$ is here a continuum variable, while in the previous section it was a site on the honeycomb lattice. The bare gauge field propagator is

$$
W_{\mu \nu}(\mathbf{x})=\frac{1}{2} \int \frac{d^{3} \mathbf{q}}{(2 \pi)^{3}} \frac{e^{i \mathbf{q} x}}{|\mathbf{q}|}\left(\Pi_{\mu \nu}(\mathbf{q})+\beta \frac{\mathbf{q}_{\nu} \mathbf{q}_{\mu}}{\mathbf{q}^{2}}\right),
$$

with the usual transverse projector

$$
\Pi_{\mu \nu}(\mathbf{q})=\delta_{\mu \nu}-\frac{\mathbf{q}_{\mu} \mathbf{q}_{v}}{\mathbf{q}^{2}}
$$

The above field theory is closely related to the threedimensional quantum electrodynamics $\left(\mathrm{QED}_{3}\right),{ }^{16}$ with one important caveat: the "Maxwell" term is now already at the bare level nonanalytic in momentum, and proportional to $|\mathbf{q}|$, and not to the usual $\mathbf{q}^{2}$. It can be obtained from the reduced quantum electrodynamics $\mathrm{QED}_{4,3}$ in which the electromagnetic fields live in $3+1$ dimensions, but are coupled to fermions which are confined to the lower, $(2+1)$-dimensional "brane," by "integrating out" the out-of-plane components of the vector potential. ${ }^{17}$ This procedure in general also changes the effective gauge-fixing parameter from $\beta^{\prime}$ in the original $(3+1)$-dimensional theory into $\beta$ in the above expression, as in $\beta=\left(1+\beta^{\prime}\right) / 2$. We see that only the Feynman gauge $(\beta=1)$ remains invariant under this dimensional reduction, which makes it the most convenient one from the practical point of view. The nonanalyticity of the gauge field propagator around $\mathbf{q}=0$ can be understood as the reason for the exact marginality of the charge coupling. ${ }^{18}$

Fermions appear quadratically in the action and can be (formally) integrated out. If we redefine the fields as $e a_{\mu} \rightarrow a_{\mu}$, and $e A_{\mu} \rightarrow A_{\mu}$, and then shift the fluctuating field as $a_{\mu}-A_{\mu} \rightarrow a_{\mu}$, the result of this integration would be the action

$$
\widetilde{S}=\frac{1}{2} \int \frac{d \mathbf{q}}{(2 \pi)^{3}}\left[\frac{N}{8}|\mathbf{q}| \Pi_{\mu \nu}(\mathbf{q}) a_{\mu}(\mathbf{q}) a_{\nu}(-\mathbf{q})+\frac{2}{e^{2}}|\mathbf{q}|\left(\Pi_{\mu \nu}(\mathbf{q})+\frac{\mathbf{q}_{\nu} \mathbf{q}_{\mu}}{\beta \mathbf{q}^{2}}\right)\left[a_{\mu}(\mathbf{q})+A_{\mu}(\mathbf{q})\right]\left[a_{v}(-\mathbf{q})+A_{\nu}(-\mathbf{q})\right]\right]+V(a) .
$$

The first term proportional to $N$ is the familiar one-loop polarization in the $\mathrm{QED}_{3},{ }^{19}$ and the second term is the quasi-Maxwell term, which now after the shift of variables also includes the external probe; $V(a)$ is a sum of monomials in the fluctuating gauge field $a$ with degree $\geqslant 4$. Note that $V(a)$ does not contain the external probe $A_{\mu}$, which after the shift appears only in the quasi-Maxwell term. This allows one to differentiate with respect to the external probe, and so to obtain the current-current correlation function in terms of the exact gauge field propagator, $D_{\mu \nu}^{a a}=\left\langle a_{\mu} a_{\nu}\right\rangle$ :

$$
\left\langle j_{\mu}(\mathbf{q}) j_{v}(-\mathbf{q})\right\rangle=\frac{2}{e^{2}}|\mathbf{q}|\left(\Pi_{\mu \nu}(\mathbf{q})+\frac{1}{\beta} \frac{\mathbf{q}_{v} \mathbf{q}_{\mu}}{\mathbf{q}^{2}}\right)-\frac{2}{e^{2}}|\mathbf{q}|\left(\Pi_{\mu \alpha}(\mathbf{q})+\frac{1}{\beta} \frac{\mathbf{q}_{\mu} \mathbf{q}_{\alpha}}{\mathbf{q}^{2}}\right) D_{\alpha \beta}^{a a}(\mathbf{q}) \frac{2}{e^{2}}|\mathbf{q}|\left(\Pi_{\beta \nu}(\mathbf{q})+\frac{1}{\beta} \frac{\mathbf{q}_{\beta} \mathbf{q}_{v}}{\mathbf{q}^{2}}\right) .
$$

Current conservation, on the other hand, dictates that the exact gauge-field propagator has the form ${ }^{20}$

$$
D_{\mu \nu}^{a a}(\mathbf{q})=\frac{1}{|\mathbf{q}|}\left(R \Pi_{\mu \nu}(\mathbf{q})+\beta \frac{e^{2}}{2} \frac{\mathbf{q}_{\nu} \mathbf{q}_{\mu}}{\mathbf{q}^{2}}\right),
$$

where $R$ is a function of the number of fermions $N$ and of the coupling $e^{2}$. Inserting this form into the expression for the current-current correlation function, we find that

$$
\left\langle j_{\mu}(\mathbf{q}) j_{\nu}(-\mathbf{q})\right\rangle=\sigma|\mathbf{q}| \Pi_{\mu \nu}(\mathbf{q}),
$$

where the optical conductivity $\sigma$, in units of $e^{2} / \hbar$, and in the limits $T=0$ and $\omega \rightarrow 0$, is simply

$$
\sigma=\frac{2}{e^{2}}\left(1-\frac{2 R}{e^{2}}\right) \text {. }
$$

Note that the current-current correlation function is completely independent of the gauge-fixing parameter $\beta$, just as one expects. We can rewrite (22) as a perturbed Gaussian action

$$
\begin{aligned}
\widetilde{S}= & \frac{1}{2} \int \frac{d \mathbf{q}}{(2 \pi)^{3}}\left\{\left(\frac{N}{8}+\frac{2}{e^{2}}\right)|\mathbf{q}|\left(\Pi_{\mu \nu}(\mathbf{q})+\gamma \frac{\mathbf{q}_{\nu} \mathbf{q}_{\mu}}{\mathbf{q}^{2}}\right) a_{\mu}(\mathbf{q}) a_{\nu}(\mathbf{q})+\frac{2}{e^{2}}|\mathbf{q}|\left(\Pi_{\mu \nu}(\mathbf{q})+\frac{1}{\beta} \frac{\mathbf{q}_{\nu} \mathbf{q}_{\mu}}{q^{2}}\right)\right. \\
& \left.\times\left[2 a_{\mu}(\mathbf{q}) A_{\nu}(-\mathbf{q})+A_{\mu}(\mathbf{q}) A_{\nu}(-\mathbf{q})\right]\right\}+V(a),
\end{aligned}
$$

where $\gamma=\left(2 / \beta e^{2}\right)\left[1 /\left(N / 8+2 / e^{2}\right)\right]$. In the Gaussian approximation, that is neglecting the higher-order terms given by
$V(a)$, the functional integral can be explicitly performed and the constant $R$ is given by the value $R_{0}=\left[(N / 8)+\left(2 / e^{2}\right)\right]^{-1}$. 
From (26) this way we find the Ioffe-Larkin-like ${ }^{21}$ result for the conductivity,

$$
\sigma^{-1}=\left[\frac{2}{e^{2}}\left(1-R_{0} \frac{2}{e^{2}}\right)\right]^{-1}=\frac{8}{N}+\frac{e^{2}}{2},
$$

which can be interpreted as the addition of the fermion's and the gauge-field's resistivities into the total resistivity. Expanding to the first power in the weak charge coupling $e^{2}$ yields $\sigma=(N / 8)\left[1-\left(N e^{2} / 16\right)+\cdots\right]$, in agreement with the lattice computation.

To go beyond the Gaussian approximation we can expand in powers of the effective charge in the theory $e^{2} /\left[2+\left(N e^{2} / 8\right)\right]$; one finds

$$
\frac{1}{R}=\frac{2}{e^{2}}+\frac{N}{8}+N x \frac{e^{2}}{\frac{N}{8} e^{2}+2} \cdots,
$$

where $x=\left(92-9 \pi^{2}\right) /\left[(4 \pi)^{2} 18\right] .^{22}$ In the weak coupling regime $e^{2} \ll 1 / N$, after expanding in powers of $e^{2}$,

$$
\begin{aligned}
\sigma & =\frac{2}{e^{2}}\left(1-\frac{1}{1+\frac{N}{16} e^{2}+\frac{N x e^{4}}{4}+\cdots}\right) \\
& =\frac{N}{8}\left(1-\frac{N e^{2}}{16}+e^{2} 4 x+O\left(e^{4}\right) \cdots\right),
\end{aligned}
$$

so that Eq. (1) is recovered, with the numerical value of the constant

$$
C_{1}=8 x=\frac{23}{(3 \pi)^{2}}-\frac{1}{4}=0.0089319 .
$$

On the other hand, in the large- $N$ limit, $N \gg 1 / e^{2}$, we expand in powers of $1 / N$,

$$
\sigma=\frac{2}{e^{2}}\left(1-\frac{2}{e^{2}} \frac{1}{\frac{N}{8}+\frac{2}{e^{2}}+8 x\left(1-\frac{16}{N e^{2}}\right)+\cdots}\right),
$$

so that

$$
\sigma=\frac{2}{e^{2}}\left(1-\frac{16}{N e^{2}}+\frac{128}{N^{2} e^{4}}\left(2+C_{1} e^{2}\right)+O\left(N^{-3}\right)\right) .
$$

\section{CONCLUSION}

In conclusion, we have presented the computation of the universal zero temperature, low frequency (optical) conductivity in the ultimate, relativistic, low-energy regime in graphene, when the Fermi velocity has reached the velocity of light. Although this regime lies beyond presently available experimental conditions, such as the temperature and the sample sizes, the issue of ultimate value of the optical conductivity is theoretically interesting, and presents an important question of principle. We find that the ultimate value of the conductivity is universal, and dependent only on the fine-structure constant of the media surrounding the graphene sheet, but in principle different from the noninteracting value seen in the experiment. The difference from the noninteracting value is of the order of the fine structure constant itself, however, and therefore of the relative size of the order of $1 \%$.

\section{ACKNOWLEDGMENT}

I.F.H. was supported by the NSERC of Canada.
${ }^{1}$ R. R. Nair, P. Blake, A. N. Grigorenko, K. S. Novoselov, T. J. Booth, T. Stauber, N. M. R. Peres, and A. K. Geim, Science 320, 1308 (2008).

${ }^{2}$ A. W. W. Ludwig, M. P. A. Fisher, R. Shankar, and G. Grinstein, Phys. Rev. B 50, 7526 (1994).

${ }^{3}$ A. Giuliani, V. Mastropietro, and M. Porta, Phys. Rev. B 83, 195401 (2011); Commun. Math. Phys. 311, 317 (2012).

${ }^{4}$ V. Juričić, O. Vafek, and I. F. Herbut, Phys. Rev. B 82, 235402 (2010); I. F. Herbut, V. Juričić, O. Vafek, and M. J. Case, arXiv:0809.0725.

${ }^{5}$ I. F. Herbut, V. Juričić, and O. Vafek, Phys. Rev. Lett. 100, 046403 (2008).

${ }^{6}$ J. González, F. Guinea, and M. A. H. Vozmediano, Phys. Rev. B 59, R2474 (1999).

${ }^{7}$ E. G. Mishchenko, Europhys. Lett. 83, 17005 (2008).

${ }^{8}$ D. E. Sheehy and J. Schmalian, Phys. Rev. B 80, 193411 (2009).

${ }^{9}$ I. Sodemann and M. M. Fogler, Phys. Rev. B 86, 115408 (2012).

${ }^{10}$ B. Rosenstein, M. Lewkowicz, and T. Maniv, Phys. Rev. Lett. 110, 066602 (2013).
${ }^{11}$ J. González, F. Guinea, and M. A. H. Vozmediano, Nucl. Phys. B 424, 595 (1994).

${ }^{12}$ A. Giuliani, V. Mastropietro, and M. Porta, Phys. Rev. B 82, 121418 (2010); Ann. Phys. (NY) 327, 461 (2012).

${ }^{13}$ C. L. Kane and E. J. Mele, Phys. Rev. Lett. 95, 226801 (2005).

${ }^{14}$ A. Giuliani and V. Mastropietro, Phys. Rev. B 85, 045420 (2012).

${ }^{15}$ I. F. Herbut, Phys. Rev. Lett. 79, 3502 (1997); 81, 3916 (1998).

${ }^{16}$ R. D. Pisarski, Phys. Rev. D 29, 2423 (1984); T. W. Appelquist, D. Nash, and L. C. R. Wijewardhana, Phys. Rev. Lett. 60, 2575 (1988).

${ }^{17}$ E. V. Gorbar, V. P. Gusynin, and V. A. Miransky, Phys. Rev. D 64, 105028 (2001)

${ }^{18}$ I. F. Herbut, Phys. Rev. Lett. 87, 137004 (2001).

${ }^{19}$ See I. F. Herbut, Phys. Rev. Lett. 88, 047006 (2002); Phys. Rev. B 66, 094504 (2002), and references therein.

${ }^{20}$ I. F. Herbut and Z. Tešanović, Phys. Rev. Lett. 76, 4588 (1996); 78, 980 (1997).

${ }^{21}$ I. F. Herbut, Phys. Rev. Lett. 94, 237001 (2005).

${ }^{22}$ S. Teber, Phys. Rev. D 86, 025005 (2012), particularly Eq. (45a). 